Article

\title{
Neisseria mucosa does not inhibit the growth of Neisseria gonor- rhoeae
}

Saïd Abdellati ${ }^{1 \$}$, Jolein Laumen ${ }^{1,2 \$}$, Natalia Gonzalez ${ }^{1}$, Sheeba S. Manoharan-Basil ${ }^{1}$, Christophe Van Dijck ${ }^{1,2}$, Tessa De Block ${ }^{1}$, Irith De Baetselier ${ }^{1}$, Delphine Martiny ${ }^{3}$, Chris Kenyon ${ }^{1,4}$

1 HIV/STI Unit, Institute of Tropical Medicine, Antwerp, Belgium; ckenyon@itg.be

2 University of Antwerp, Laboratory of Medical Microbiology, Vaccine and Infectious disease Institute, Antwerp, Belgium

3 Laboratoire Hospitalier Universitaire de Bruxelles

4 Division of Infectious Diseases and HIV Medicine, University of Cape Town, Anzio Road, Observatory 7700, South Africa; ckenyon@itg.be

* Correspondence: ckenyon@itg.be

$\$$ These two authors contributed equally to this publication

Abstract: Antibiotic-sparing treatments are required to prevent the further emergence of antimicrobial resistance in Neisseria gonorrhoeae. Commensal Neisseria species have previously been found to inhibit the growth of pathogenic Neisseria species. For example, a previous study found that 3 out of 5 historical isolates of Neisseria mucosacould inhibit the growth of $N$. gonorrhoeae. In this study, we used agar overlay assays to assess if 24 circulating and historical isolates of Neisseria mucosa could inhibit the growth of 28 circulating and historical isolates of $N$. gonorrhoeae. Although pitting around each colony of $N$. mucosa created an optical illusion of decreased growth of $N$. gonorrhoeae, we found no evidence of inhibition $(n=24)$. In contrast, positive controls of Streptococcus pneumoniae and Escherichia colidemonstrated a strong inhibitory effect against the growth of $N$. gonorrhoeae.

Keywords: Neisseria mucosa; Neisseria gonorrhoeae; agar overlay assay; bacterial competition

\section{Introduction}

A number of countries worldwide are reporting an increasing incidence of sexually transmitted infections due to Neisseria gonorrhoeae (1). This combined with increasing antimicrobial resistance in this organism has led to efforts to find novel therapies to treat and prevent this infection (2). One of these strategies has been to use antiseptics to prevent acquisition and transmission of $N$. gonorrhoeae to and from the oropharynx $(2,3)$. The prevalence of $N$. gonorrhoeae in the pharynx may reach $10 \%$ in high-risk populations and $N$. gonorrhoeae has been shown to be highly susceptible in vitro to antiseptics such as Listerine (3-6). A pilot clinical study found that a Listerine mouthwash reduced the prevalence of pharyngeal $N$. gonorrhoeae as assessed by culture (5). These findings provided the motivation for two randomized controlled trials that assessed if Listerine could reduce the incidence of $N$. gonorrhoeae and other STIs in men who have sex with men $(2,3)$.

One of these was the preventing resistance in gonorrhoea (PReGo) study conducted in our centre [2]. This placebo-controlled trial randomized high-risk men who have sex 
with men to intensive use of Listerine ${ }^{\circledR}$ mouthwash and gargle or placebo to try to reduce the incidence of bacterial STIs in this population. The study found that Listerine increased rather than decreased the incidence of oropharyngeal $N$. gonorrhoeae. Listerine ${ }^{\circledR}$ had a similar though statistically non-significant effect in the other study that used a slightly different study design (the OMEGA study) [6]. One of the possible explanations for these surprising results is that Listerine could reduce the abundance of commensal bacteria that have an inhibitory effect on $N$. gonorrhoeae. One such commensal bacteria is Neisseria mucosa, which has recently been shown to inhibit the growth of $N$. gonorrhoeae by Aho et al (7).

$N$. mucosa is a healthy core component of the oropharyngeal microbiome and even low concentrations of Listerine ${ }^{\circledR}$ have been shown to be bacteriocidal to Neisseria spp [7]. If the use of the Listerine ${ }^{\circledR}$ mouthwash reduced the prevalence/abundance of $N$. mucosa and N. mucosa inhibits the growth of $N$. gonorrhoeae, then Listerine ${ }^{\circ}$ could increase the susceptibility for $N$. gonorrhoeae infection [2]. In a similar vein, a randomized controlled trial established that nasal inoculation with $N$. lactamica reduced the incidence of colonization with $N$. meningitidis [8]. If the in-vitro anti-gonococcal effect of $N$. mucosa could be confirmed, $N$. mucosa might be evaluated as a probiotic to prevent gonococcal infection.

This provided the justification for the current study where we aimed to test if our locally circulating isolates of $N$. mucosa and other commensal Neisseria, including those circulating in the PReGo participants, were able to inhibit the growth of $N$. gonorrhoeae.

\section{Results}

Agar overlay assays were used to assess if 24 circulating and historical isolates of Neisseria mucosa and 16 isolates from other Neisseria species could inhibit the growth of 28 circulating and historical isolates of $N$. gonorrhoeae.

None of the commensal Neisseria or N. meningitidis exhibited any activity against $N$. gonorrhoeae (Table 1). The isolate of $S$. pneumoniae demonstrated clear evidence of inhibition against all 9 strains of $N$. gonorrhoeae (median diameter of inhibition $=21 \mathrm{~mm}$ ) (Figure $1 \mathrm{a}$ and $\mathrm{b}$ ). The inhibitory effect of $E$. coliwas less pronounced (Figure $1 \mathrm{a}$ and b). Inhibition was evident in 3 out of $9 \mathrm{~N}$. gonorrhoeae strains tested median diameter of inhibition $11 \mathrm{~mm}$ (Table 1).

A proportion of the colonies of $N$. mucosa exhibited a repellant effect, whereby they repelled the layer of agar poured over them (Figure 1c and d). This created 'pitting colonies or a convex slope between the top of the second layer of agar and the edge of each $N$. mucosa colony, which created an illusion of reduced $N$. gonorrhoeae growth around each $N$. mucosa colony [15] (Figure 2). Closer visual inspection, however, confirmed that $N$. gonorrhoeae growth over this convex slope around the $N$. mucosa colonies was not macroscopically distinguishable from that elsewhere (Figure $1 \mathrm{c}$ and d). 
Table 1. Inhibitory activity of various commensal Neisseria and other species in agar overlay assay
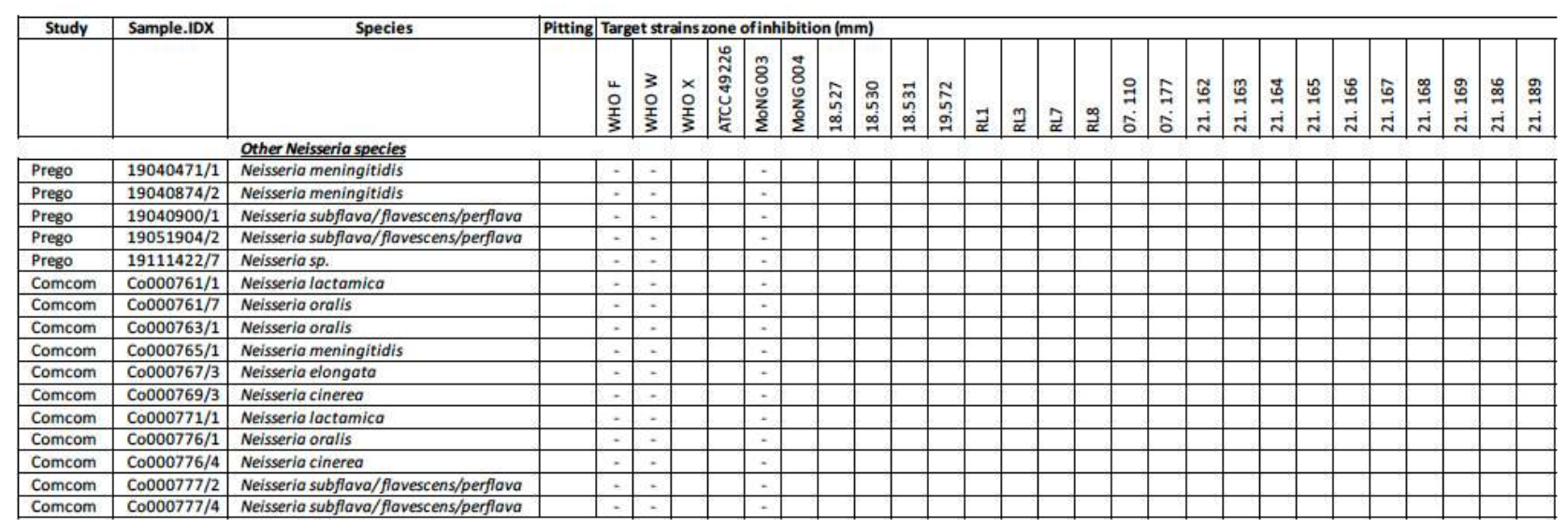

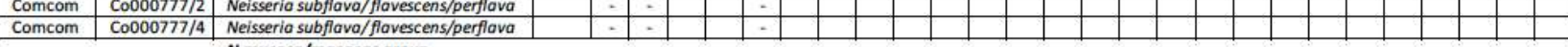

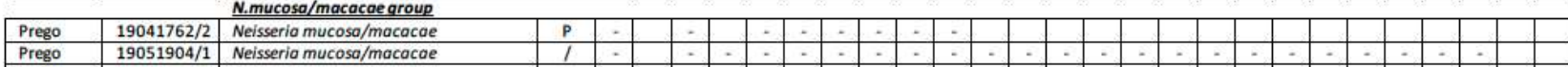

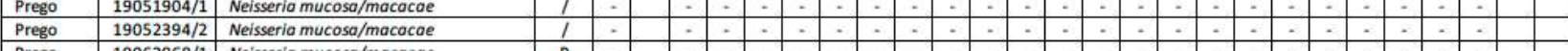

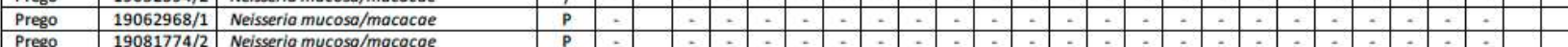

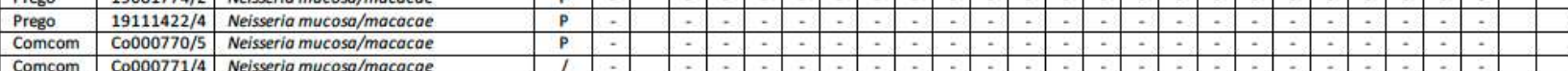

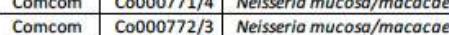

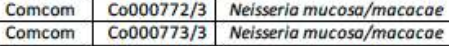

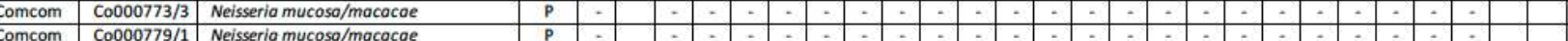

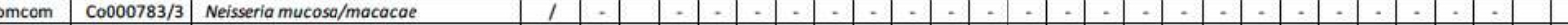

Comcom 1 Co000787/3 Neisserio mucoso/maccacoe

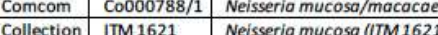

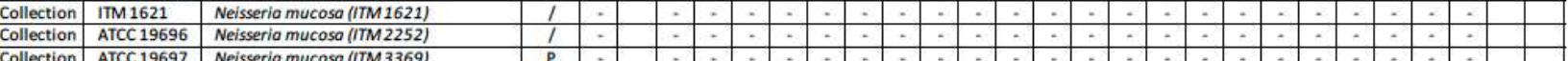
Collection ATCC19695 Neiseria mucoso (17M3375)

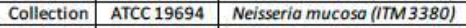

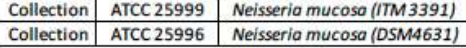

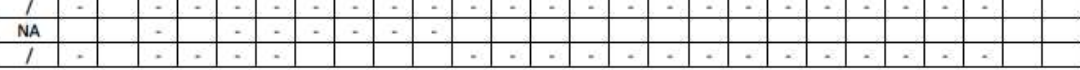

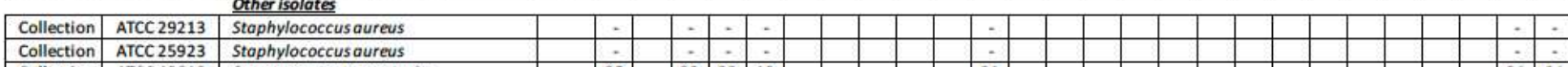

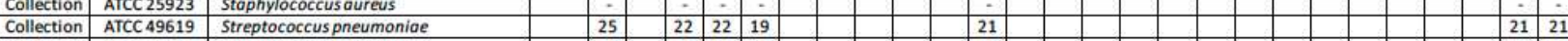

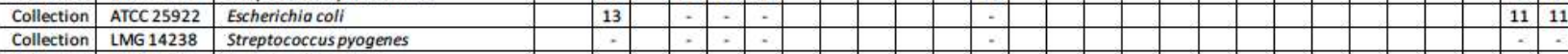

Co inhibition, NA: Not available, P.pitting observed, /: no pitting observed 
Figure 1. Agar overlay assay testing the ability of various bacterial species to inhibit the growth of a lawn of N. gonorrhoeae strain RL1 (a) and strain 21.189 (b). Only the colonies of Escherichia coli $(\mathrm{Ec})$ and Streptococcus pneumoniae (Sp) inhibit the growth. The colonies of N. mucosa in (c) and (d) do not exhibit any inhibitory effect on the growth of $N$. gonorrhoeae strain 21.163 (c) and strain WHO-W (d). A close up of one of the N. mucosa colonies in (d) demonstrates the pitting $(p)$ of the upper layer of agar around the right-hand colony of $N$. mucosa.

a

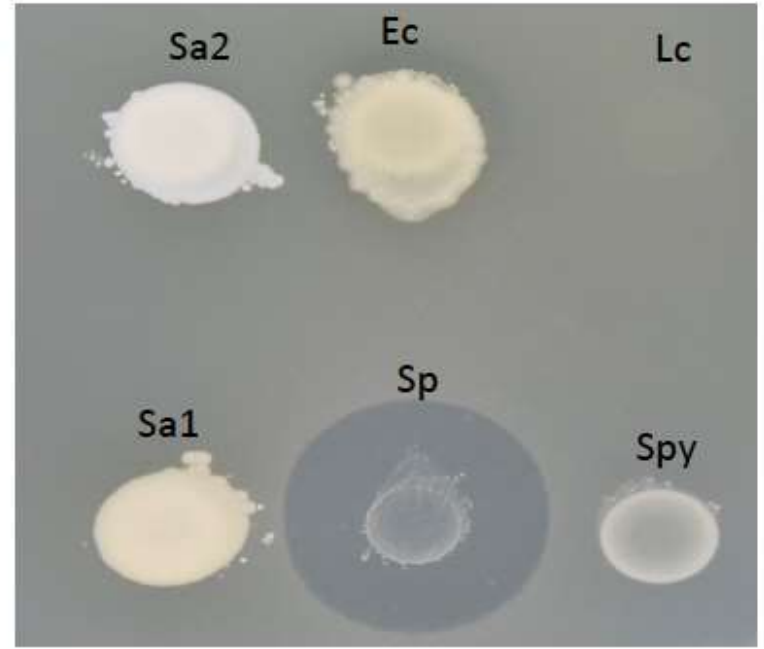

c

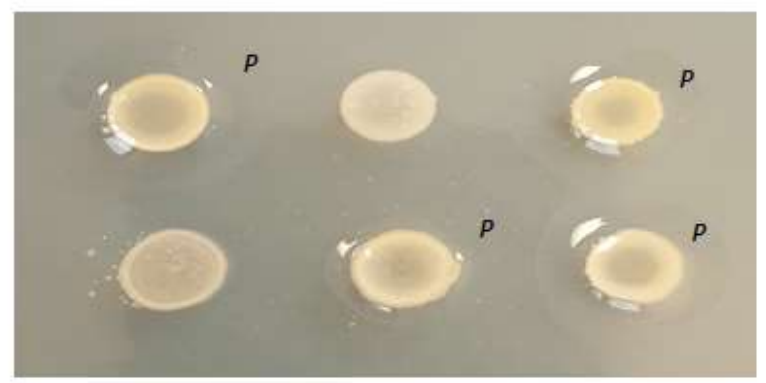

b

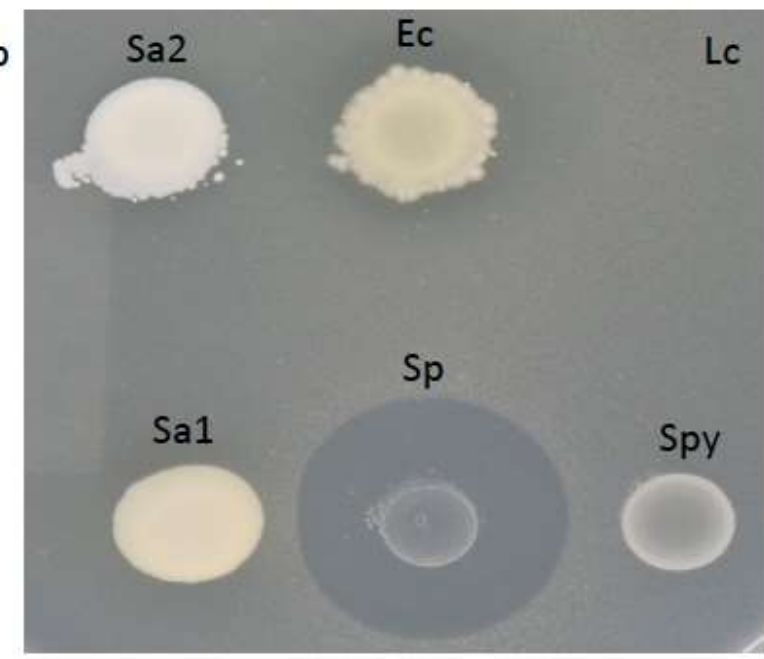

d

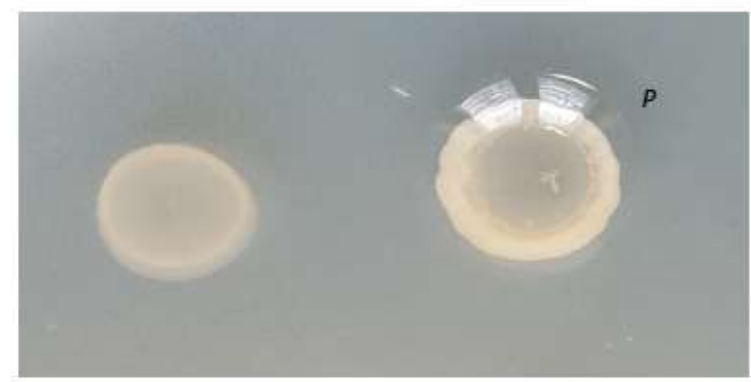

Ec - Escherichia coli (ATCC 25922); Lc - Lactobacillus crispatus (LMG 9479); p - pitting; Sa - Staphylococcus aureus (1:ATCC 29213, 2:ATCC 25913); Sp - Streptococcus pneumoniae (ATCC 49619); Spy - Streptococcus pyogenes (LMG 14238). 


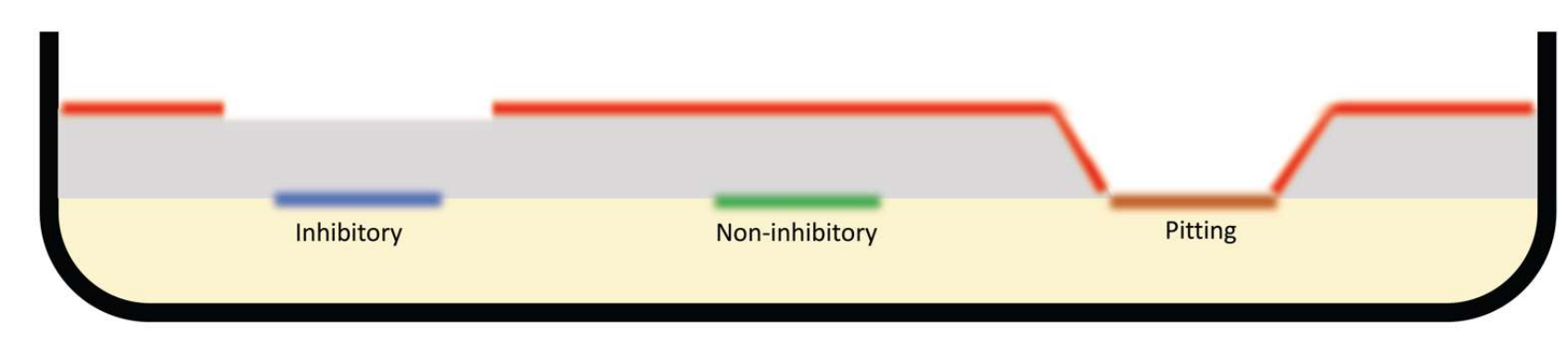

Figure 2. A schematic illustration of the difference between growth-inhibition and pitting in the agar overlay assay. An agar plate in cross section is depicted, on which 3 bacterial colonies (labeled 'inhibitory', 'non-inhibitory' and 'pitting') have been spotted and incubated for 24 hours before a layer of GCB agar containing $10^{6} \mathrm{CFU} / \mathrm{ml}$ of $N$. gonorrhoeae is poured over the plate (grey layer). Whilst the 'non-inhibitory' colony has no effect on the growth of $N$. gonorrhoeae (red line), and the 'inhibitory' colony has a clear inhibitory effect, the major effect of the 'pitting' colony is to repel the second layer of agar thus creating an area around it which appears more translucent from above. Close visual inspection, including from the lateral aspect, of the depressed sections of the second layer of agar around the 'pitting' colony reveal uninhibited growth of $N$. gonorrhoeae. 


\section{Discussion}

Unlike Aho et al., we could find no evidence that $N$. mucosa or any other commensal Neisseria was able to inhibit the growth of $N$. gonorrhoeae [1]. This was despite using a large number of clinical and reference strains of $N$. gonorrhoeae as target strains, and the largest collection of commensal Neisseria tested to date as inhibitory bacteria.

How can these discordant findings be explained? Aho found this inhibitory effect in 3 out of $5 \mathrm{~N}$. mucosa isolates. The isolates were all obtained from ATCC collections and did not include any recent clinical isolates. No photos were provided of the agar overlay assays showing that $N$. mucosa inhibited the growth of $N$. gonorrhoeae. However, one image of $N$. mucosa inhibiting the growth of $N$. flavescens was provided.

In our study, we followed an identical agar overlay protocol utilizing a larger panel of isolates of $N$. mucosa and $N$. gonorrhoeae. The experiments were performed by a laboratory technician with over 25 years of experience culturing Neisseria species (SA). The plates were examined by this person and two others with extensive experience in culturing Neisseria species (CK and JL). All three concurred that pitting around each colony of $N$. mucosacreated an optical illusion of decreased growth around the colony. Close visual inspection confirmed that there was no inhibition of growth.

We consider this a parsimonious explanation for the different findings between the two studies. It could be possible that only certain strains of $N$. mucosa are able to inhibit specific strains of $N$. gonorrhoeae and that we did not include any of these combinations in our experiments. We did, however, test one of the three isolates of $N$. mucosa shown to have an inhibitory effect by Aho et al. This isolate (ATCC 25996) had no effect on the growth of 23 contemporarily circulating strains of $N$. gonorrhoeae in our laboratory. We did not have access to, and therefore did not include any of the same strains of $N$. gonorrhoeae used by Aho et al. As a result, we cannot exclude the possibility that our N. mucosa strains would have had an inhibitory effect on the $N$. gonorrhoeae strains used by Aho et al. Furthermore, our experiments were not conducted in duplicate. In pilot studies we found that $N$. mucosae did not inhibit the growth of $N$. gonorrhoeae and our experiment was thus designed to maximize the chances of detecting any inhibitory effect on $N$. gonorrhoeae. We thus evaluated if any strains of $N$. mucosa we could access $(n=24)$ could inhibit the growth of a large panel of strains of circulating and type strains of $N$. gonorrhoeae $(n=28)$. This constitutes the largest experiment to have assessed this effect. The previous largest experiment was conducted with 4 isolates of $N$. mucosa tested against one isolate of $N$. gonorrhoeae and a further one isolate of $N$. mucosa tested against 7 isolates of $N$. gonorrhoeae (1). Because we found no evidence of inhibition in any of the pair-wise comparisons in our experiments, we consider it unlikely that repeating the experiments in triplicate would change our findings.

We mainly included pharyngeal $N$. gonorrhoeae target strains. Since these were isolated from asymptomatic individuals, they may have adapted to live with oral commensals. Therefore, a greater number of strains from anatomical sites other than the pharynx should be included in future studies. We also cannot completely exclude the possibility that an unevaluated different experimental condition such as storage of the isolates or the source of the agar used was responsible for the differences in the results between the two studies. It could be argued that a further weakness of the study is that inhibition was only assessed 
via visual inspection. This is however the standard method of assessing growth inhibition in the agar overlay assay (1). Our study, unlike that of Aho et al., did include positive controls. These showed clear and consistent evidence of inhibition. Taken together, these findings suggest that $N$. mucosa is unlikely to have a significant inhibitory effect on the growth of N. gonorrhoeae - at least in the agar overlay assays evaluated here. More importantly for our current research, we consider it unlikely that a broad range of $\mathrm{N}$. mucosa isolates contains a sufficiently potent compound against our currently circulating strains of $N$. gonorrhoeae to be able to explain the findings of the PReGo and OMEGA studies.

We concur with Aho et al., that commensal microbes represent a possible source of antimicrobial compounds that could play an important role in reducing the emergence of AMR in N. gonorrhoeae and other bacteria. Based on our findings, we consider it more likely that such anti-gonococcal compounds will be discovered from organisms such as $S$. pneumoniae than N. mucosa $[12,16]$.

\section{Materials and Methods}

\subsection{Origin of bacterial isolates}

4.1.1. Inhibitory/producer bacterial isolates

Most Neisseria isolates were obtained from two clinical studies conducted at our centre:

i) The Preventing Resistance in Gonorrhoea Study (PReGo), a single center randomized controlled trial conducted at the Institute of Tropical Medicine in Antwerp, Belgium, between 2019 and 2020 that assessed the efficacy of an antiseptic mouthwash to prevent STIs among 343 MSM using PrEP [2].

ii) The Commensals in the Community Study (ComCom), a survey of the oropharyngeal microbiomes of Institute of Tropical Medicine (ITM) employees conducted in June

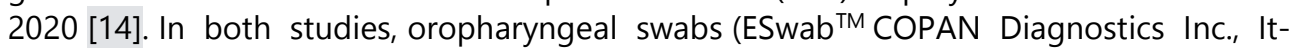
aly) were taken and inoculated onto blood and Modified Thayer-Martin agar plates using the streak plate technique and incubated at $35-37^{\circ} \mathrm{C}$ and $5 \% \mathrm{CO}_{2}$. Plates were examined after 48 hours, and Neisseria-like colonies were selected based on a positive Oxidase test and a Gram stain. Neisseria-like colonies were enriched on blood agar plates and stored in skim milk at $-80^{\circ} \mathrm{C}$. Cultures of Neisseria-like colonies were shipped to Laboratoire des Hôpitaux Universitaires de Bruxelles-Universitair Laboratorium Brussel (LHUB-ULB) where species were identified using Matrix-Assisted Laser Desorption/lonization Time-of-Flight Mass Spectrometry (MALDI-TOF MS), on a MALDI Biotyper ${ }^{\circ}$ Sirius IVD system using the MBT Compass IVD software and library (Bruker Daltonics, Bremen, Germany) consisting of 9607 spectra.

All N. mucosa isolates obtained from the PReGo and ComCom studies $(n=14)$ as well as a random selection of $\mathrm{N}$. meningitidis $(\mathrm{n}=3)$ and other commensal Neisseria obtained from these two studies - N. subflava $(n=4), N$. cinerea $(n=2), N$. lactamica $(n=1), N$. oralis $(n=3), N$. elongata $(n=1) \quad$ and Neisseria spp. $\quad(n=1 n=1 n=1$; Table 1) were included in the present work.

In addition, we also included 6 N. mucosa isolates from our ITM historical collection. Five of these were ATCC strains and one was a historical clinical specimen obtained from a patient in 1977 and the DSM4631/ATCC 25996 isolate used by Aho et al., was obtained from the DSMZ (https://www.dsmz.de/collection/catalogue/details/culture/DSM-46).

\subsubsection{N. gonorrhoeae target strains}


Three strains of $N$. gonorrhoeae were used as target strains for all experiments (WHO$\mathrm{F}, \mathrm{WHO}-\mathrm{X}$ and $\mathrm{MoNgO03}$ - a clinical isolate obtained from an individual with asymptomatic pharyngeal N. gonorrhoeae infection attending our STI clinic in 2020. In addition, one ATCC strain of $N$. gonorrhoeae, WHO-W and 23 other circulating strains of $N$. gonorrhoeae were tested against some of the putative inhibitory bacteria (Table 1).

\subsubsection{Non-Neisseria isolates}

ATCC strains of Streptococcus pneumoniae $(\mathrm{n}=1 ; \mathrm{ATCC}$ 49619) and Escherichia coli $(n=1$; ATCC 25922) were used as positive controls for the agar overlay inhibition tests. We also assessed the inhibitory effects of three other bacterial species obtained from ATCC: Staphylococcus aureus ( $\mathrm{n}=2$; ATCC 29213, ATCC 25913), Streptococcus pyogenes $(n=1$; LMG 14238) and Lactobacillus crispatus ( $n=1$; LMG 9479).

\subsection{Agar overlay assay}

The details of the agar overlay assay have been described elsewhere [1]. Briefly, all strains used in the experiment were propagated on Columbian blood agar plates for 18$24 \mathrm{~h}$. The cultures were suspended in $10 \mu \mathrm{l}$ of phosphate-buffered saline (PBS) containing $10^{9} \mathrm{CFU} / \mathrm{ml}$ of inhibitory strains. These were spotted onto GC agar and incubated in $5 \% \mathrm{CO}_{2}$ at $35-37^{\circ} \mathrm{C}$ for $24 \mathrm{~h} .10 \mathrm{ml}$ of melted GCB agar containing $10^{6} \mathrm{CFU} / \mathrm{ml}$ of a target strain was added to each spotted plate. The plates were then re-incubated for 24 to 48 hours. The diameter of the zone of inhibition surrounding each producer strain was assessed at 24 hours.

Author Contributions: Conceptualization, CK, SA and JL.; methodology, SA.; software, CK; validation, SA, JL; formal analysis, SA, CK.; investigation, SA.; writing-original draft preparation, CK.; writingreview and editing, CK.; visualization, SA, CK.; supervision, CK; All authors have read and agreed to the published version of the manuscript.

Funding: This research received no external funding

Institutional Review Board Statement: Ethics approval was obtained from ITM's Institutional Review Board (1276/18 and 1351/20) and from the Ethics Committee of the University of Antwerp $(19 / 06 / 058$ and $A B / a c / 003)$.

Informed Consent Statement: Not applicable.

Data Availability Statement: All the relevant data generated during this study is provided in table 1

Acknowledgments: We would like to thank the PReGo and ComCom study participants for providing the samples used in this study.

Conflicts of Interest: The authors declare no conflict of interest.

References initial manuscript

1. Aho EL, Ogle JM, Finck AM. The Human Microbiome as a Focus of Antibiotic Discovery: Neisseria mucosa Displays Activity Against Neisseria gonorrhoeae. Front Microbiol. 2020;11. doi: ARTN 577762 10.3389/fmicb.2020.577762. PubMed PMID: WOS:000599309800001.

2. Van Dijck C, Tsoumanis A, Rotsaert A, Vuylsteke B, Van den Bossche D, Paeleman E, et al. Antibacterial mouthwash to prevent sexually transmitted infections in men who have sex with men taking HIV pre-exposure prophylaxis (PReGo): a randomised, placebo-controlled, crossover trial. The Lancet Infectious Diseases. 2021;21(5):657- 67.

3. Wong ML, Chan RK, Koh D, Wee S. Increase in oral sex and pharyngeal gonorrhoea: an unintended effect of a successful condom promotion programme for vaginal sex. AIDS. 1999;13(14):1981-2. PubMed PMID: 10513660. 
4. Centers for Disease C, Prevention. Transmission of primary and secondary syphilis by oral sex--Chicago, Illinois, 1998-2002. MMWR Morb Mortal Wkly Rep. 2004;53(41):966-8. Epub 2004/10/22. PubMed PMID: 15496825.

5. Barbee LA, Khosropour CM, Dombrowski JC, Manhart LE, Golden MR. An estimate of the proportion of symptomatic gonococcal, chlamydial and non-gonococcal non-chlamydial urethritis attributable to oral sex among men who have sex with men: a case-control study. Sex Transm Infect. 2016;92(2):155-60. Epub 2015/08/25. doi: 10.1136/sextrans-2015-052214. PubMed PMID: 26297719; PubMed Central PMCID: PMCPMC4861816.

6. Chow EP, Williamson DA, Hocking JS, Law MG, Maddaford K, Bradshaw CS, et al. Antiseptic mouthwash for gonorrhoea prevention (OMEGA): a randomised, double-blind, parallel-group, multicentre trial. The Lancet Infectious Diseases. 2021;21(5):647-56.

7. Laumen J.G.E., Abedllati S, De Baetselier I, Crucitti T, Xavier B.B., Chapelle S., et al. Mechanisms of increased resistance to chlorhexidine following exposure of Neisseria gonorrhoeae isolates to chlorhexidine. ECCMID 2021. 2021.

8. Deasy AM, Guccione E, Dale AP, Andrews N, Evans CM, Bennett JS, et al. Nasal Inoculation of the Commensal Neisseria lactamica Inhibits Carriage of Neisseria meningitidis by Young Adults: A Controlled Human Infection Study. Clin Infect Dis. 2015;60(10):1512-20. Epub 2015/03/31. doi: 10.1093/cid/civ098. PubMed PMID: 25814628.

9. Graver MA, Wade JJ. The role of acidification in the inhibition of Neisseria gonorrhoeae by vaginal lactobacilli during anaerobic growth. Ann Clin Microb Anti. 2011;10. doi: Artn 8 10.1186/1476-0711-10-8. PubMed PMID: WOS:000208655000008.

10. Bouttier S, Yeo A, Any-Grah AASA, Geiger S, Huang N, Nicolas V, et al. Characterization and in vitro evaluation of a vaginal gel containing Lactobacillus crispatus for the prevention of gonorrhea. Int J Pharmaceut. 2020;588. doi: ARTN 225 119733 226 10.1016/j.ijpharm.2020.119733. PubMed PMID: WOS:000577558900001.

11. Bisaillon JG, Beaudet R, Saheb SA, Morisset R. Interference of Neisseria gonorrhoeae growth by aerobic bacterial representatives of the urogenital flora. Rev Can Biol. 1980;39(4):201-8. Epub 1980/12/01. PubMed PMID: 6785845.

12. McBride ME, Duncan WC, Knox JM. Bacterial interference of Neisseria gonorrhoeae by alpha-haemolytic streptococci. Br J Vener Dis. 1980;56(4):235-8. Epub 1980/08/01. doi: 10.1136/sti.56.4.235. PubMed PMID: 6775764; PubMed Central PMCID: PMCPMC1045785.

13. Kraus SJ, Geller RC, Perkins GH, Rhoden DL. Interference by Neisseria gonorrhoeae growth by other bacterial 233 species. J Clin Microbiol. 1976;4(3):288-95. Epub 1976/09/01. doi: 10.1128/jcm.4.3.288-295.1976. PubMed PMID: 234 823175; PubMed Central PMCID: PMCPMC274452.

14. Laumen JGE, Van Dijck C, Abdellati S, De Baetselier I, Manoharan-Basil SS, Van den Bossche D, et al. Low Macrolide and Fluoroquinolone Susceptibility in Non-pathogenic Neisseriae in the General Belgian Population and STI 237 Clinic Attendees. IMID 4-6 Nov 2021. 2021

15. Kandi V. Bacterial Colony Characters: Pitting Colonies. Journal of Medical Microbiology \& Diagnosis. 2015;4(2):1.

16. Lux T, Nuhn M, Hakenbeck R, Reichmann P. Diversity of bacteriocins and activity spectrum in Streptococcus pneumoniae. J Bacteriol. 2007;189(21):7741-51. Epub 2007/08/21. doi: 10.1128/JB.00474-07. PubMed PMID: 17704229; 241 PubMed Central PMCID: PMCPMC2168751.1.

References (new in revised version: between () in text)

1. Unemo M, Bradshaw CS, Hocking JS, de Vries HJC, Francis SC, Mabey D, et al. Sexually transmitted infections: challenges ahead. Lancet Infect Dis [Internet]. 2017;17(8):e235-79. Available from: http://dx.doi.org/10.1016/S1473-3099(17)30310-9

2. Van Dijck C, Tsoumanis A, Rotsaert A, Vuylsteke B, Van den Bossche D, Paeleman E, et al. Antibacterial mouthwash to prevent sexually transmitted infections in men who have sex with men taking HIV pre-exposure prophylaxis (PReGo): a randomised, placebo-controlled, crossover trial. Lancet Infect Dis [Internet]. 2021;21(5):657-67. Available from: http://dx.doi.org/10.1016/S1473-3099(20)30778-7

3. Chow EPF, Williamson DA, Hocking JS, Law MG, Maddaford K, Bradshaw CS, et al. Antiseptic mouthwash for 
gonorrhoea prevention (OMEGA): a randomised, double-blind, parallel-group, multicentre trial. Lancet Infect Dis [Internet]. 2021;21(5):647-56. Available from: http://dx.doi.org/10.1016/S1473-3099(20)30704-0

4. Van Dijck C, Kenyon C, Cuylaerts V, Sollie P, Spychala A, De Baetselier I, et al. The development of mouthwashes without anti-gonococcal activity for controlled clinical trials: An in vitro study. F1000Research. 2020;8:1-11.

5. Chow EPF, Howden BP, Walker S, Lee D, Bradshaw CS, Chen MY, et al. Antiseptic mouthwash against pharyngeal Neisseria gonorrhoeae: A randomised controlled trial and an in vitro study. Sex Transm Infect. 2017;93(2):88-93.

6. Tsoumanis A, Hens N, Kenyon CR. Is Screening for Chlamydia and Gonorrhea in Men Who Have Sex with Men Associated with Reduction of the Prevalence of these Infections? A Systematic Review of Observational Studies. Sex Transm Dis. 2018;45(9):615-22.

7. Aho EL, Ogle JM, Finck AM. The Human Microbiome as a Focus of Antibiotic Discovery: Neisseria mucosa Displays Activity Against Neisseria gonorrhoeae. Front Microbiol. 2020;11(December):1-8. 